\title{
Crossing Numbers of Graphs, Lower Bound Techniques and Algorithms: A Survey
}

\author{
Farhad Shahrokhi` László A. Székely ${ }^{\star \star ~ I m r i c h ~ V r t ' o \star \star \star ~}$
}

\begin{abstract}
We give a survey of recent techniques for deriving lower bounds and algorithms for constructing upper bounds for several variations of the crossing number problem. Our aim is to emphasize the more general results or those results which have an algorithmic flavor, including the recent results of the authors.
\end{abstract}

\section{Introduction}

Topological graph theory is a beautiful and profound subject in which most problems are conceptually intricate and computationally intractable. Among the problems in this field, the problem of drawing graphs with minimum number of edge crossings in the plane has been studied extensively by mathematicians $[1,4,9,15,19,20,31]$, and also by the computer science and the VLSI communities $[21,7]$. Some very effective methods for deriving lower bounds on the planar crossing number were obtained by this second group and has remained unknown to the graph theory community. For instance, a recently published paper in graph theory [22] announced the lower bound of $\Omega\left(k^{c \log k}\right)$ for the crossing number of the $k$-dimensional cube, where the lower bound of $\Omega\left(4^{k}\right)$ is easily achievable by standard VLSI techniques. In particular, the only existing algorithm for computing provably near-optimal solutions to the planar crossing number problem has been provided by the VLSI community [7].

There have not been too many results dealing with the crossing number problem on a surface, perhaps due to the difficulty of the problem which had made the existing tools inadequate. There had been some very elegant and valuable results published in this area by the mathematics community $[14,18,16,17,18,23]$. However, these results are either stated for selected family of graphs, or if they are general they do not appear to be effective for some specific cases. In particular, the methods have been ad-hoc and did not provide for a computationally effective approach.

Very recently, a computationally effective method which could provide for a provably near-optimal number of crossings on surfaces has been discovered $[25,27]$. This method also provides for a general upper bound on the number of crossings which quantifies the extent to which the addition of a handle or crosscap can be useful. Moreover, the speculations on how to minimize the number of edge crossings when drawing graphs on the books, has led to the discovery of the first computationally effective method which provides for provably near-optimal drawings of graphs with straight lines in the plane [28]. The method also provides for an upper bound on the number crossings in the straight line drawing which involves the planar crossing number. Thus, the extent to which drawing the edges with straight lines would increase the number of crossings has been quantified.

* Department of Computer Science, University of North Texas P.O.Box 13886, Denton, TX, 76203, USA

** Department of Computer Science, Eötvös University 1088 Budapest, Muzeum krt. 6-8, Hungary

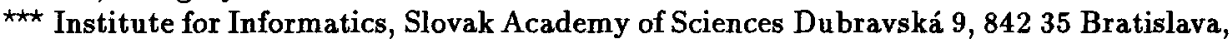
Slovak Republic. Research of this author was partially supported by Grant No. 88 of Slovak Academy of Sciences and by EC Cooperative action IC1000 "Algorithms for Future Technologies" (Project ALTEC) 
In deriving the results in $[25,27,28]$ we have adopted and generalized the tools provided by the VLSI community $[21,7]$. We were also inspired and guided by the previous results obtained by the graph theorists $[1,18,19,16,17]$.

In this paper we will survey the methods for deriving general lower bounds, and algorithms for constructing upper bounds for several variations of the crossing number problems. Our intention is to mention the results which have been obtained by the two groups and would be of interest to both the computer scientists and the mathematicians. However, we would only explicitly mention those methods which are either very general, or have some algorithmic flavor, including our own results. Nevertheless, we have tried to include most of the important results related to the crossing number problems in our bibliography. Section 2 contains the basic definitions. Section 3 contains the methods for deriving the lower bounds, whereas Section 4 contains the algorithms and the methods for deriving the upper bounds.

\section{Basic Definitions}

Throughout this paper we assume $G=(V, E)$ has $n$ vertices, $m$ edges, maximum degree $\Delta$ and minimum degree $\delta$. Let $d_{i}(G)$ denote the degree of vertex $i \in V$. (For concepts of topological graph theory not defined here in detail, see [31].) For $g \geq 0$, let $S_{g}$ and $N_{g}$ denote the orientable surface and non-orientable surface, respectively, of genus $g$. Thus $S_{g}$ is topologically equivalent to a sphere with $g$ handles (or holes) and $N_{g}$ is topologically equivalent to a sphere with $g$ crosscaps. To get a feeling about these surfaces, note that $S_{1}$ is the torus, $S_{2}$ is the double torus, $N_{1}$ is the real projective plane, and $N_{2}$ is the Klein bottle. It is well known that any compact 2-manifold is topologically equivalent to either $S_{g}$ or $N_{g}$. Throughout this paper a surface means a compact 2-manifold.

A drawing $D$ of a graph $G$ on a surface $S$ consists of placing the vertices of $G$ on $S$ and drawing the edges of $G$ using the continuous curves of $S$ between the corresponding vertices, such that no curve has a vertex as an internal point and no point is an internal point of 3 curves.

Let $c r_{g}(G)$ and $\tilde{c r}_{g}(G)$ denote the minimum number of crossings over all drawings of $G$ on $S_{g}$ and $N_{g}$, respectively. We call $c r_{g}(G)$ and $\tilde{c}_{g}(G)$, the crossing number of $G$ on $S_{g}$ and $N_{g}$, respectively. Note that $c r_{0}(G)$ is the familiar planar crossing number. Let $c \bar{r}_{0}(G)$ denote the the minimum number of edge crossings when each edge of $G$ is drawn by exactly one straight line segment in the plane. $c \bar{r}_{0}(G)$ is called the rectilinear crossing number of $G$.

For a drawing $D$ of $G$ on a surface with $g$ handles or crosscaps, let $\mathrm{cr}_{g}(D)$ denote the number of edge crossings in $D$.

For any partition of the vertex set $V$ of $G$ into two disjoint parts $V_{1}$ and $V_{2}$, let $E\left(V_{1}, V_{2}\right)$ denote the set of edges with one endpoint in $V_{1}$ and the other in $V_{2}$. Define the bisection width of $G$ as

$$
b(G)=\min _{\left|V_{1}\right|,\left|V_{2}\right| \geq n / 3}\left\{\left|E\left(V_{1}, V_{2}\right)\right|\right\}
$$

where the minimum is taken over all partitions $V=V_{1} \cup V_{2}$ with $\left|V_{1}\right|,\left|V_{2}\right| \geq n / 3$. Also, define $\vec{b}(G)=\max _{H \leq G} b(H)$, where the maximum is taken overall subgraphs of $G$. 
A book consists of a spine and $k$ pages, $k \geq 1$. The spine of the book is a line. Each page is a half-plane that has the spine as its boundary. Informally, a drawing of an undirected graph $G=(V, E)$ in the book consists of an ordering of the vertices on the spine and then drawing each edge of the graph in one page of the book with a curve, such that, any curve has only its two end-points on the spine and no three curves intersect in one point unless it is an end-point in common. Let us denote by $\nu_{k}(G)$ the minimum number of crossings among all $k$-page book embedding of $G$. For a drawing $D$ of $G$ on a $k$-page book, let $\nu_{k}(D)$ denote the number of edge crossings in $D$.

Let $G_{1}=<V_{1}, E_{1}>$ and $G_{2}=<V_{2}, E_{2}>$ be two undirected graphs, $\left|V_{1}\right| \leq\left|V_{2}\right|$. An embedding of $G_{1}$ in $G_{2}$ is a pair of mappings $\omega=\langle\phi, \psi\rangle$ satisfying

$$
\begin{gathered}
\phi: V_{1} \rightarrow V_{2} \text { is an injection } \\
\left.\psi: E_{1} \rightarrow \text { set of all paths in } G_{2}\right\},
\end{gathered}
$$

such that if $u v \in E_{1}$ then $\psi(u v)$ is a path between $\phi(u)$ and $\phi(v)$. For any $e \in E_{2}$ and any $u \in V_{2}$ define

$$
\begin{aligned}
\mu_{\omega}(e) & =\left|\left\{f \in E_{1}: e \in \psi(f)\right\}\right| \text { and } \\
m_{\omega}(u) & =\left|\left\{f \in E_{1}: u \in \psi(f)\right\}\right|
\end{aligned}
$$

Moreover, we define,

$$
\begin{aligned}
\mu_{\omega} & =\max _{e \in E_{2}} \mu_{\omega}(e), \text { and } \\
m_{\omega} & =\max _{u \in V_{2}} m_{\omega}(u) .
\end{aligned}
$$

We refer to $\mu_{\omega}$ and $m_{\omega}$ as the edge congestion and vertex congestion of $\omega$, respectively.

\section{The Lower Bounds}

\subsection{Euler's Formula, Induction and Counting}

Using the Euler's polyhedral formula it is easy to see that for any graph $G=$ $(V, E)$, with $|V|=n,|E|=m$

$$
\operatorname{cr}_{0}(G) \geq m-3 n+6 .
$$

The generalizations of this result on a surface are due to Kainen [18] and Kainen and White [19].

Theorem 1. Let $G=(V, E)$ be a graph without loops and parallel edges with $|V|=n,|E|=m$. Let $l, l \geq 3$ be the length of the smallest cycle in $G$. Then

$$
\begin{gathered}
c r_{g}(G) \geq m-\frac{l}{l-2}(n-2+2 g) \\
c \tilde{r}_{g}(G) \geq m-\frac{l}{l-2}(n-2+g)
\end{gathered}
$$


Proof. We demonstrate the orientable case only. The nonorientable case is similar. Suppose $G$ is drawn on $S_{g}$ with $\operatorname{cr}_{g}(G)$ crossings. We choose a minimal set $E_{0}$ of edges whose removal permit $G$ to be embedded in $S_{g}$. Then

$$
c r_{g}(G) \geq\left|E_{0}\right| \text {. }
$$

This is easily seen by choosing for each crossing, an edge which is involved in the crossing. Denote $G^{\prime}=G-E_{0}$. Then $c r_{g}\left(G^{\prime}\right)=0$. Clearly $\gamma\left(G^{\prime}\right) \leq g$. Let $G^{\prime}$ is embedded in $S_{g}$. Then $G^{\prime}$ is a 2-cell embedding in $S_{g}$, that is, $S_{g}-G^{\prime}$ is a collection of open discs, or faces. The boundary of each face is a cycle in $G^{\prime}$. Suppose each cycle is bounded by at least $l^{\prime}$ edges. Note that $l^{\prime} \geq l$. Therefore $l^{\prime} f \leq 2\left(m-\left|E_{0}\right|\right)$, where $f$ is the number of faces. This together with Euler's formula for $G^{\prime} n-\left(m-\left|E_{0}\right|\right)+f=2-2 \gamma\left(G^{\prime}\right)$ easily implies

$$
\left|E_{0}\right| \geq m-\frac{l^{\prime}}{l^{\prime}-2}\left(n-2+2 \gamma\left(G^{\prime}\right)\right) \geq m-\frac{l}{l-2}(n-2+2 g) .
$$

Noting (2) we get the claimed result.

Theorem 1 by itself may not provide for strong lower bounds, and it is often useful to combine it with other methods.

The first general lower bound for $\operatorname{cr}_{0}(G)$ has been derived by Ajtai et al. [1], and, independently by Leighton [21]. The method used to obtain the lower bound combines the Euler's formula (on the plane) with induction.

Theorem 2. [1, 21] For any $G$, with $m \geq 4 n$ one has,

$$
c r_{0}(G) \geq \frac{m^{3}}{100 n^{2}}
$$

Proof sketch. We show by induction on $n$ that for $m \geq 4 n$

$$
\operatorname{cr}_{0}(G) \geq \frac{3}{64}\left(\begin{array}{l}
n \\
4
\end{array}\right) \frac{m^{3}}{\left(\begin{array}{l}
n \\
2
\end{array}\right)^{3}}
$$

Note that for $n \leq 9$, the inequality $m \geq 4 n$ can only satisfied for $K_{9}$ with $n=9$ and $m=36$, in which case the result follows from 1 . For $n \geq 10$, if $5 n \geq m \geq 4 n$, then the claim follows from (1) since the right-hand side of (3) is at most $m-3 n$. If $m>5 n$, then note that

$$
(n-4) c r_{0}(G) \geq \sum_{x \in V} c r_{0}\left(G_{x}\right)
$$

where $G_{x}$ is a graph obtained by removing vertex $x$ from $G$. Note that the number of edges of $G_{x}$, denoted by $m_{x}$ is $m-d_{x} \geq 4 n$, and thus the induction hypothesis applies to $G_{x}$. Consequently,

$$
\sum_{x \in V} c r_{0}\left(G_{x}\right) \geq \frac{3}{64}\left(\begin{array}{c}
n-1 \\
4
\end{array}\right) \frac{\sum_{x \in V} m_{x}^{3}}{\left(\begin{array}{c}
n-1 \\
2
\end{array}\right)^{3}} .
$$

Now (3) follows easily by doing some algebra. To complete the proof observe that for $n \geq 9$, the right-hand side of (3) is at least $m^{3} / 100 n^{2}$.

We now point out the analogue to Theorem 2 on a surface which is derived using the counting method. The counting method, normally requires to inspect the subgraphs of a desirable size (or structure) and apply the lower bounds in Theorem 1 to the subgraphs. The counting argument in very special cases, where $G$ is $K_{n}$ or $K_{m, n}$, and $g=0,1$, was used previously in [16, 17, 20]. 
Theorem 3. [27] Let $G=(V, E)$, with $m \geq 8 n$. Let $D$ be any drawing of $G$ on a surface of genus $g$. Then the following hold.

$$
\begin{gathered}
c r_{g}(D)=\Omega\left(\frac{m^{3}}{n^{2}}\right), \text { provided that } n^{2} / m>g \geq 0, \\
c r_{g}(D)=\Omega\left(\frac{m^{2}}{g}\right), \text { provided that } n^{2} / m \leq g \leq m / 64 .
\end{gathered}
$$

Proof. We prove the orientable case only. The proof for the nonorientable case is similar. Let $r, 4 \leq r \leq n$ be an integer. Let $G^{\prime}=\left(V^{\prime}, E^{\prime}\right)$ be an induced subgraph of $G$ with $\left|V^{\prime}\right|=r$ and $D^{\prime}$ be a subdrawing in $D$ associated with $G^{\prime}$. By Theorem 1 , we must have, $\operatorname{cr}_{g}\left(D^{\prime}\right) \geq\left|E^{\prime}\right|-3(r+2 g)$. Summing up these inequalities over all drawings associated with induced subgraphs $G^{\prime}$ with $r$ vertices, we obtain

$$
\sum_{\substack{\sigma^{\prime}=\left(V^{\prime}, E^{\prime}\right) \\
\left|V^{\prime}\right|=r}} c r_{g}\left(D^{\prime}\right) \geq m\left(\begin{array}{l}
n-2 \\
r-2
\end{array}\right)-3(r+2 g)\left(\begin{array}{l}
n \\
r
\end{array}\right) .
$$

Moreover, observe that

$$
\left(\begin{array}{l}
n-4 \\
r-4
\end{array}\right) \operatorname{cr}_{g}(D)=\sum_{\substack{\sigma^{\prime}=<V^{\prime}, E^{\prime}>\\
\left|V^{\prime}\right|=r}} c r_{g}\left(D^{\prime}\right)
$$

which implies

$$
\begin{aligned}
c r_{g}(D) & \geq \frac{(n-2)(n-3)}{(r-2)(r-3)}\left(m-3(r+2 g) \frac{n(n-1)}{r(r-1)}\right) \\
& \geq \frac{(n-2)(n-3)}{(r-2)(r-3)}\left(m-6(r+2 g) \frac{n^{2}}{r^{2}}\right),
\end{aligned}
$$

where the last inequality is implied by $r \geq 4$. We try to set the value of $r$ to get the best possible lower bound. If $0 \leq g \leq n^{2} / m$, then, we set $r=\left\lceil 8 n^{2} / m\right\rceil$, note that $4 \leq r \leq n$. Observe that in this case, $6(r+2 g)\left(n^{2} / r^{2}\right) \leq\left(6 n^{2} / r\right)+\left(12 g n^{2} / r^{2}\right) \leq$ $60 m / 64$, since $r \geq 8 n^{2} / m$ and $g<n^{2} / m$. Using (4), we get $c r_{g}(D) \geq m^{3} / 1300 n^{2}$. Now assume that $n^{2} / m \leq g \leq m / 64$, set $r=\left\lceil 8 \sqrt{g n^{2} / m}\right\rceil$, and note that $4 \leq r \leq n$. Moreover, in this case $r \leq 8 g$, since $n^{2} / m \leq g$, and consequently we have, $6(r+2 g)\left(n^{2} / r^{2}\right) \leq\left(60 g n^{2} / r^{2}\right) \leq 60 m / 64$, since $r \geq 8 \sqrt{g n^{2} / m}$. Now (4) implies $c r_{g}(D) \geq m^{2} / 463 g$.

Let $n=o(m)$ and observe that the lower bound in Theorem 2 dominates the lower bound in (1). Similarly, for $n=o(m)$, the lower bound in Theorem 3 dominate the lower bounds of Theorem 1.

Another application of the counting method can be demonstrated by deriving a lower bound on $\nu_{k}(G)$.

Theorem 4. [28] For any $G$ with $n$ vertices, $m$ edges, where $m \geq 3 n k$,

$$
\nu_{k}(G) \geq \frac{m^{3}}{37 k^{2} n^{2}}-\frac{27 k n}{37} .
$$




\subsection{The Bisection Method}

The bisection method applies effectively when $G$ is drawn on the plane or on a surface. The method starts with inserting vertices of degree 4 at the edge crossings and then applying a suitable separator theorem.

The following result of Leighton [21] which is proved using the planar separator theorem, has some nice algorithmic implications in approximating the planar crossing number.

Theorem 5. [21] For any graph $G$ of bounded degree,

$$
c r_{0}(G)+n=\Omega\left(b(G)^{2}\right) .
$$

Proof. Consider a drawing of $G$ with $c r_{0}(G)$ crossings. Introducing a new vertex at each crossing, we obtain a plane graph $H$ with $N=n+c r_{0}(G)$ vertices. Assign weight 0 to each new vertex and weight of $1 / \mathrm{n}$ to all other vertices. Since, $G$ has bounded degree, $H$ has also bounded degree, thus, it follows by the application of the planar separator theorem that, by deletion of at most

$$
O\left(\sqrt{n+c r_{0}(G)}\right)
$$

edges, $H$ can be separated into two parts $H_{1}=\left(V_{H_{1}}, E_{H_{1}}\right)$ and $H_{2}=\left(V_{H_{2}}, E_{H_{2}}\right)$ such that the sets $V_{1}=V_{H_{1}} \cap V$ and $V_{2}=V_{H_{2}} \cap V$ have at least $n / 3$ elements. Hence

$$
b(G) \leq O\left(\sqrt{n+c r_{0}(G)}\right)
$$

and the result follows.

A more general version of Theorem 5 can be shown using the separator theorem of Gazit and Miller [13] and is known among VLSI community; a detailed proof can be found in [30] or [24].

Theorem 6. Let $G=(V, E)$ and $d_{i}(G), i \in V$ be degrees of vertices of $G$ then

$$
c r_{0}(G)+\sum_{i \in V} d_{i}^{2}(G)=\Omega\left(b(G)^{2}\right) .
$$

Sykora and Vrt'o [29] have shown that any $G$ of genus at most $g$ has a bisection which is at most $5 \sqrt{3 n(g+1) \Delta}$. Using this result they obtained the following lower bound on a surface.

Theorem 7. [29] For any graph $G$ with $n$ vertices and maximal degree $\Delta$

$$
\operatorname{cr}_{g}(G) \geq \frac{b^{2}(G)}{75(g+1) \Delta}-n .
$$

\subsection{The Embedding Method}

Leighton [21] used the concept of graph embedding and introduced a technique for deriving lower bounds on the planar crossing number of the perfect shuffle and the mesh of trees. His technique requires an estimate for congestion of embedding $K_{n}$ into the underlying graph $G$. Shahrokhi and Székely [26] generalized Leighton's approach to graphs with unbounded degrees and gave estimates of embedding $K_{n}$ into a large class of symmetric graphs. For instance the following result was shown. 
Theorem 8. [26] Let $G$ be an edge transitive graph with $n \geq 65$ and $m \leq$ $n(n-1) / 22 \log n$. Then

$$
c r_{0}(G) \geq \frac{1}{45}\left(\frac{n m}{\tilde{d}}\right)^{2}(1-o(1))-\frac{n}{2} \Delta,
$$

where $\bar{d}$ is the average distance in $G$. Moreover, if $m=o\left(\frac{n^{2}}{\log n}\right)$, then $\frac{1}{45}$ can be replaced by $\frac{1}{20}$.

Let $Q_{t}$ denote the $t$-dimensional cube. Theorem 8 gives

$$
c r_{0}\left(Q_{t}\right) \geq \frac{1}{20} 4^{t}(1-o(1))
$$

which is the best known lower bound previously obtained by Sýkora and Vrt'o using an ad-hoc method.

Further generalization was obtained by Shahrokhi, Sýkora, Székely and Vrt'o [25], [27] who showed the following result.

Theorem 9. [25, 27] Let $G=(V, E)$ and $H=\left(V^{\prime}, E^{\prime}\right)$ be graphs with $\left|V^{\prime}\right| \leq$ $|V|=n$ and let $\omega$ be an embedding of $H$ into $G$. Let $D_{G}$ be a drawing of $G$ on a surface $S_{g}$ of genus $g$. Then there is a drawing $D_{H}$ of $H$ on $S_{g}$ such that

$$
c r_{g}\left(D_{G}\right) \geq \frac{c r_{g}\left(D_{H}\right)}{\mu_{\omega}^{2}}-\frac{n}{2}\left(\frac{m_{\omega}}{\mu_{\omega}}\right)^{2}
$$

Proof sketch. Consider an embedding $\omega=(\phi, \psi)$ of $H$ into $G$ with edge congestion $\mu_{\omega}$ and vertex congestion $m_{\omega}$. Without loss of generality we assume that small neighborhoods of the vertices are disks and that the continuous curves representing the edges of $G$ in the drawing $D_{G}$ turn into straight line segments in the disks. Using $D_{G}$ and the embedding of $H$ into $G$, we shall obtain a drawing $D_{H}$ of $H$ on $S_{g}$. The vertex set will be the same $V$. For an edge $e=i j$ of $H$, let $p=\psi(e)$ be the active $\phi(i) \phi(j)$ path in $G$ and $p^{D_{G}}$ be the drawing of $p$ in $D_{G}$. To draw the edge $e$ on $S_{g}$, we draw a curve along $p^{D_{G}}$ between the vertices $\phi(i)$ and $\phi(j)$, which does not pass through any intermediate vertex of $p$. We assume (with no loss of generality) that this curve of $D_{2}$ goes "parallel" with $p^{D_{\sigma}}$, and also very close to $p^{D_{G}}$. It can be shown that the total number of crossings in $D_{H}$ is at most $\operatorname{cr}_{g}\left(D_{G}\right) \mu_{\omega}^{2}+n m_{\omega}^{2} / 2$. We have, therefore

$$
n m_{\omega}^{2} / 2+c r_{g}\left(D_{G}\right) \mu_{\omega}^{2} \geq c r_{g}\left(D_{H}\right)
$$

which implies the result.

The application of Theorem 9 requires an embedding of $H$ in $G$ with suitable values for edge and vertex congestion. Note that we can take $D_{H}$ to be an optimal drawing of $H$ on $S_{g}$, and get a lower bound for $\operatorname{cr}_{g}\left(D_{G}\right)$ for any drawing $D_{G}$ of $G$. Conversely, if $D_{G}$ is given, then we can a drawing $D_{H}$ which satisfies the inequality. When applying Theorem $9, H$ is normally taken to be a complete graph.

Let $g=o\left(\frac{2^{t}}{t^{3}}\right)$, then the application of Theorem 9 gives

$$
c r_{g}\left(Q_{t}\right)=\Omega\left(\frac{4^{t}}{g}\right)
$$


which is asymptotically stronger than the lower bounds obtained by the applications of Theorems 3 and 7. For larger values of $g$, say $2^{t-4} t \geq g \geq 2^{t-1}$, Theorem 3 gives

$$
c r_{g}\left(Q_{t}\right)=\Omega\left(\frac{4^{t} t^{2}}{g}\right)
$$

which asymptotically dominates the lower bounds obtained by the applications of Theorems 7 and 9.

\section{Algorithms}

\subsection{A Divide and Conquer Algorithm}

Computing $c r_{0}(G)$ is known to be NP-hard [12], thus it only make sense to approximate it. There is one algorithm which can provide provably near-optimal solution for the planar crossing number, when $G$ has bounded degree. This goes back to Bhatt and Leighton [7]. For the special case of bipartite graphs, when the locations of vertices in one partite set are fixed on the plane, very efficient algorithms which produce constant times optimal solutions have been recently discovered by Eades and Wormald [10].

Theorem 10. [7] Suppose that there is polynomial time algorithm which approximates $b(G)$ to within a multiplicative factor of $R(n)$, for any $n$ vertex graph $G$, where $R(n)$ is some nondecreasing functional measure of error. Then for any degree bounded graph $G$, there is a polynomial time algorithm which produces a drawing of $G$ in the plane with at most

$$
\left.O\left(c r_{0}(G)+n\right) R^{2}(n) \log ^{2} n\right)
$$

edge crossings.

In proving the above results Bhatt and Leighton recursively bisect $G$, to $G_{1}$ and $G_{2}$, and draw the edges in the cut after $G_{1}$ and $G_{2}$ are drawn. To draw an edge $e=i j$ in the cut, Bhatt and Leighton used Jordan curves which connect $i$ and $j$ to the boundary of the drawings for $G_{1}$ and $G_{2}$. These curves may have many bends, and thus in the drawings obtained through Theorem 10 the edges are not straight lines. Recently [28], the method of Theorem 10 was adopted to obtain the drawings on one page which are easily extended to $k$ pages. Moreover, these one page drawings easily give rise to straight line drawings of $G$ in the plane, and hence provide for the first provably good approximation algorithm for the rectilinear crossing number.

Theorem 11. [28] Assume that we have an approximation algorithm for bisecting any graph $G$ so that the number of edges in the cut is at most $R(n) b(G)$, where $R(n)$ is some nondecreasing functional measure of error. Then we can produce a drawing of $G$ on a one page book with at most

$$
O\left(\log ^{2} n R^{2}(n)\left(c r_{0}(G)+\sum_{i \in V} d_{i}^{2}(G)\right)\right)
$$

crossings. 
Proof sketch. Using our approximation algorithm, we recursively partition $G$, to $G_{1}$ and $G_{2}$, draw $G_{1}$ and $G_{2}$ on the one page book such that all vertices of $G_{1}$ precede all vertices of $G_{2}$ in the spine, and then insert and draw any edge $x y$ in the partition using a half circle whose diameter equals to the distance of $a$ from $b$ on the line. The process of recursively partitioning $G$ gives rise to a binary tree $T$, the partition tree of $G$, whose vertices are subgraphs of $G$. Let $H$ be a node of $T$; we denote by $H_{1}$ and $H_{2}$ the children of $H$ in $T$. Let $l^{D_{H}}$ be the maximum number of half circles that enclose any vertex in the drawing $D^{H}$ of $H$ obtained by the algorithm. It is easy to verify that,

$$
l^{D_{H}}=O(\log n R(n) \bar{b}(H)) .
$$

Moreover, it is easy to verify that

$$
\nu_{1}\left(D_{H}\right) \leq \nu_{1}\left(D_{H_{1}}\right)+\nu_{1}\left(D_{H_{2}}\right)+R^{2}(n) b^{2}(H)+\left(l^{D_{H_{1}}}+l^{D_{H_{2}}}\right)(R(n) b(H))
$$

and hence by (5) we get

$$
\nu_{1}\left(D_{H}\right) \leq \nu_{1}\left(D_{H_{1}}\right)+\nu_{1}\left(D_{H_{2}}\right)+O\left(R^{2}(n) \bar{b}^{2}(H) \log n\right) .
$$

Now one can use Theorem 6 , to solve the above recurrence relation over a subtree of $T$ rooted at $H$. We get,

$$
\nu_{1}\left(D_{H}\right)=O\left(\log ^{2} n R^{2}(n)\left(c r_{0}(H)+\sum_{i \in V_{H}} d_{i}^{2}(H)\right)\right) .
$$

This finishes the proof, since we can take $H$ to be $G$.

Very recently [8] polynomially time bounded algorithm for approximately computing the bisection within a constant multiplicative factor from the optimal were discovered. Hence, we can assume $R(n)=O(1)$ and derive the following result.

Theorem 12. [28] We can draw any graph $G$ on a 1-page book in polynomial time with at most

$$
O\left(\log ^{2} n\left(c r_{0}(G)+\sum_{i \in V} d_{i}^{2}(G)\right)\right)
$$

crossings. Moreover, assume that $G$ has $\Delta(G)=O\left(\delta(G)^{1.5}\right)$ and $m \geq 3 n$, then, the number of crossings in our drawing is within a factor of $O\left(\log ^{2} n\right)$ from the optimal value

The connection between $k$-page drawings and 1-page drawings is easy to see.

Theorem 13. [28] We can draw any graph $G$ on a $k$-page book, $k \geq 2$, in polynomial time with at most

$$
O\left(\frac{\log ^{2} n}{k}\left(c r_{0}(G)+\sum_{i \in V} d_{i}^{2}(G)\right)\right)
$$

crossings. Moreover, assume that $G$ has $\Delta=O\left(\delta^{1.5}\right)$ and $m \geq 4 n$, then, for $k=2$ the number of crossings in our drawing is within a factor of $O\left(\log ^{2} n\right)$ from the optimal value.

Bienstock and Dean $[5,6]$ have extensively investigated the relationship between $c r_{0}(G)$ and $c \bar{r}_{0}(G)$ and have obtained the following. 
Theorem 14. [5] For any graph $G$, we have,

$$
c \bar{r}_{0}(G)=O\left(\Delta c r_{0}(G)^{2}\right)
$$

The results in [5] and [6] are the first general results in the literature, regarding the relationship between $c r_{0}(G)$ and $\overline{c r_{0}}(G)$. However, these results do not provide for a provably near-optimal solution in polynomial time. The significance of drawing $G$ on one page in Theorem 12 is best understood by its relevance to near-optimal drawing of graphs with straight lines.

Theorem 15. [28] We can draw any graph $G$ with straight lines on the plane in polynomial time with at most

$$
O\left(\log ^{2} n\left(c r_{0}(G)+\sum_{i \in V} d_{i}^{2}(G)\right)\right)
$$

crossings. Moreover, the number of crossings in our drawing is within a multiplicative factor of $O\left(\log ^{2} n\right)$ from the optimal value for any $G$ with $m \geq 4 n$, and $\Delta=O\left(\delta^{1.5}\right)$.

Proof sketch. Note that a 1-page drawing of $G$ is equivalent to a drawing in which the vertices of $G$ are places as the corners of a convex polygon in the plane, in the same order as them order on the straight line, and the edges of $G$ are drawn using sides or chords of the polygon. To verify the suboptimality, one can show that for $m \geq 4 n$ and $\Delta=O\left(\delta^{1.5}\right), c r_{0}(G)$ asymptotically dominates the sum of the square of degrees.

For $m \geq 4 n$ Theorem 15 implies,

$$
\overline{c r_{0}}(G)=O\left(\Delta \log ^{2} c r_{0}(G) c r_{0}(G)\right),
$$

which improves the result in [5].

As Bienstock and Dean indicated in [5] if it is required that the coordinates of vertices are integers, then the construction in Theorem 14 requires very large integers whose sizes are not bounded by a polynomial of $n$. The question posed in [5] was then, whether the rectilinear drawing in Theorem 14 can be embedded in the plane so that all coordinates are integers whose size are bounded above by a polynomial of $n$. Note that any convex polygon on $n$ vertices could be drawn in the plane so that any vertex has integer coordinates and the largest coordinate is $O\left(n^{2}\right)$. Thus, the improved straight line drawings which are obtained in Theorem 15 can be embedded in the plane so that all coordinates are integers and the largest integer is $O\left(n^{2}\right)$. Nevertheless, if $m<4 n$, the results in [28] do not work. This is not too surprising, as Bienstock has given examples of cubic graphs for which in any optimal straight line drawings in the plane, the number of bits for the largest coordinate is not bounded by any polynomial of $n$. Thus, the difficulty of constructing straight line drawings is more likely to be experienced for very sparse graphs.

\subsection{Algorithms Based on the Drawings of $K_{n}$}

Occasionally, it is useful to have a good drawing of $K_{n}$, and draw $G$ using this drawing. This method has been most effective when drawing $G$ on surfaces and books with large number of pages. 
Theorem 16. [28] For any graph $G=(V, E)$, we can construct a drawing in a $k$-page book in polynomial time, with at most

$$
\left(\frac{1}{3 k^{2}}\left(1-\frac{1}{2 k}\right)+O\left(\frac{1}{k n}\right)\right) m^{2}
$$

many crossings.

Proof Sketch. First, we draw $K_{n}$ with $O\left(\frac{n^{4}}{k^{2}}\right)$ crossings on a $k$-page book. (see [28] for details). Next, we show the existence of a suitable drawing of $G$ by randomly drawing $G$ using the drawing of $K_{n}$. Finally, to actually obtain such a drawing of $G$, one can remove the randomness from our construction in polynomial time by verifying that the expected values of the conditional probabilities can be computed in polynomial time [2].

The upper bound from Theorem 16 is asymptotically optimal at least for dense graphs i.e. $m=\theta\left(n^{2}\right)$ and $k \leq(1-\varepsilon) m / 3 n$, for $0<\varepsilon<1$, as follows from Theorem 4 . The method of Theorem 16 can also be used to draw $G$ on a surface. In fact, this is the only existing algorithm, that we are aware of, for drawing of graphs on surfaces.

Theorem 17. [27] We can draw any $G$ on a surface of genus $g \geq 2$ with

$$
O\left(\frac{m^{2} \log ^{2} g}{g}\right)
$$

crossings in polynomial time. This number of crossings is within a factor of $O\left(\log ^{2} g\right)$ from the optimal, provided that $n^{2} / m \leq g \leq m / 8$.

Proof sketch. The proof is similar to Theorem 16, except that we need a drawing of $K_{n}$ on $S$ with $O\left(\frac{n^{4} \log ^{2} g}{g}\right)$ crossings. To get such drawing of $G$ we will use Theorem 9 with $H$ being $K_{n}$ and $G$ being the cartesian product of a cube and a perfect shuffle graph with suitable dimensions. The details are tedious and can be found in [27].

\section{References}

1. Ajtai, M., Chvátal, V., Newborn, M., M., Szemerédy, E., "Crossing-free subgraphs", Annals of Discrete Mathematics 12 (1982), 9-12.

2. Alon, N., Spencer, J.H., Erdōs, P., "The Probabilistic Method", Wiley and Sons, New York, 1992.

3. Batini, C., Nardelli, E., Talamo, M., Tamassia, R., A graphtheoretic approach to aesthetic layout of information systems diagrams, in: Proc. 10th Intl. Workshop on Graph-Theoretic Concepts in Computer Science, Trauner Verlag, Berlin, 1984, 9-18.

4. Beineke, L. W., Ringeisen, R.D, On the crossing number product of cycles and graphs of order four, J. Graph Theory 4(1980), 145-155.

5. Bienstock, D., Dean, N., "New results on rectilinear crossing Numbers and Plane Embeddings", J. Graph Theory, 16 (1992), 389-398.

6. Bienstock, D., Dean, N., "Bounds on the rectilinear crossing Numbers", J. Graph Theory 17 (1993), 333-348.

7. Bhatt, S., N., Leighton, F., T., “A framework for Solving VLSI Graph Layout Problems", Journal of Computer and System Sciences 28 (1984), 300-343. 
8. Chung, F. R. K., Yau, S.T., "A near optimal algorithm for edge separators", in: Proc. 26th Annual ACM Symposium on the Theory on Computing, ACM, New York, 1994, $1-8$.

9. Erdös, P., Guy, R. P., Crossing number problems, American Mathematical Monthly 80(1973), 52-58.

10. Eades, P., Wormald, N. C., Edge crossings in drawings of bipartite graphs, Algorithmica(1994), 11, 379-403.

11. Fáry, I., "On Straight Line Representations of Graphs", Acta Univ. Szeged Sect. Sci. Math., 11, 1948, 229-233.

12. Garey, M. R., Johnson, D. S., Crossing number is NP-complete, SIAM J. Alg. Discrete Methods 4(1983), 312-316.

13. Gazit, H., Miller, G.L., Planar separators and the Euclidean norm, in: Proc. Algorithms, Intl. Symp. SIGAL'90, Lecture Notes in Computer Science 450, Springer Verlag, Berlin, 1990, 338-347.

14. Gross, J. L., On infinite family of octahedral crossing numbers, J. Graph Theory 2 (1978), 171-178.

15. Guy, R.K., A combinatorial problem, Nabla (Bull. Malayan Math. Soc.) 7 (1960), 68-72.

16. Guy, R. K., Jenkins, T., Schaer, J., The toroidal crossing number of the complete graph, J. Combinatorial Theory 4 (1968), 376-390.

17. Guy, R. K., Jenkins, T., The toroidal crossing number of $K_{m, n}, J$. Combinatorial Theory 6 (1969), 235-250.

18. Kainen, P. C., A lower bound for crossing number of graphs with applications to $K_{n}, K_{p, q}$ and $Q(d), J$. Combinatorial Theory (B) 12 (1972), 287-298.

19. Kainen, P. C., White, A. T., On stable crossing numbers, J. Graph Theory 2 (1978) 181-187.

20. Kleitman, D. J., The crossing number of $K_{5, n}, J$. Combinatorial Theory $9(1970)$, 315-323.

21. Leighton, F. T., Complexity Issues in VLSI, MIT Press, Cambridge, 1983. Malitz, S.M., "Genus $g$ Graphs have Pagenumber $O(\sqrt{g})$ ", Journal of Algorithms, 17 (1), 84-109, 1994.

22. Madej, T., Bounds for the crossing number of the n-cube, J. Graph Theory 15 (1991), 81-97.

23. Pica, G., Pisanski., T., Ventre, A.G.S., "Cartesian product of graphs and their crossing numbers, Annals of Discrete Mathematics 30(1986), 339-346.

24. Pach, J., Shahrokhi, F., Szegedy, M., Applications of crossing numbers, in: Proc. 10th Annual ACM Symposium on Computational Geometry, ACM, New York, 1994.

25. Shahrokhi, F., Sýkora, O., Székely, L. A., Vrt'o, I., The crossing number of a graph on a compact 2-manifold, Advances in Mathematics, to appear.

26. Shahrokhi, F., Székely, L. A., "Effective lower bounds for crossing number, bisection width and balanced vertex separators in terms of symmetry", in: Proc. 2nd IPCO Conf., Pittsburgh, 1992, 102-113, also to appear in Combinatorics, Probability and Computing.

27. Shahrokhi, F., Sýkora, O., Székely, L. A., Vrt'o, I., "Improved bounds for the crossing numbers on surfaces of genus $g$ ", in: Proc. 19-th Intl. Workshop on Graph-Theoretic Concepts in Computer Science WG'93, Lecture Notes in Computer Science, Springer Verlag, Berlin, 1994, 388-395, to appear in Algorithmica.

28. Shahrokhi, F., Sýkora, O., Székely, L. A., Vrt'o, I., "On Book Embeddings and Crossing Numbers", to appear in Proc. 20-th Intl. Workshop on Graph-Theoretic Concepts in Computer Science WG'94, Lecture Notes in Computer Science, Springer Verlag, Berlin, 1995.

29. Sýkora, O., Vrto, I., Edge separators for graphs of bounded genus with applications, Theoretical Computer Science 112 (1993), 419-429.

30. Sýkora, O., Vrt'o, I., On VLSI layout of the star graph and related networks, Integration, the VLSI Journal 17 (1994), 83-93.

31. White, A. T., Beineke, L. W., Topological graph theory, in: Selected Topics in Graph Theory, eds. L. W. Beineke and R. J. Wilson, Academic Press, 1978, 15-50. 